\title{
Field Testing Capabilities of the nees@UCLA Equipment Site for Soil-Structure Interaction Applications
}

\author{
Jonathan P. Stewart, ${ }^{\text {a) }}$ Daniel H. Whang, ${ }^{\text {b) }}$ and John W. Wallace ${ }^{\text {a) }}$
}

The nees@UCLA field testing has equipment for field testing and monitoring of structural and geotechnical performance. The equipment includes shakers for exciting structural and/or foundation systems, numerous sensors for monitoring accelerations and deformations within the excited structure (e.g., accelerometers, displacement transducers, strain gauges), and real time data acquisition and dissemination capabilities. A key application area for this equipment is testing of soil-foundation-structure systems. Such testing can, for example, be used to evaluate the stiffness and damping associated with foundation-soil interaction. Existing test data for such phenomena is limited, hence there is a significant need for this type of research. The results would enable the verification and calibration of computational and design models used in practice.

\section{INTRODUCTION}

The U.S. National Science Foundation is developing the George E. Brown, Jr. Network for Earthquake Engineering Simulation (NEES) Program with the goal of transforming the nation's ability to carry out earthquake engineering research. In particular, NEES seeks to shift the emphasis from current reliance on physical testing to integrated experimentation, computation, theory, databases and model-based simulation. To support this goal, 15 different advanced testing facilities, termed Equipment Sites, are being developed that will be geographically distributed across the United States. The Equipment Sites will consist of (a) structural laboratories, (b) shaking tables, (c) geotechnical centrifuges, (d) mobile and permanent field testing facilities and (e) a tsunami wave basin.

\footnotetext{
a) Associate Professor, Civil and Environmental Engineering Dept., University of California, Los Angeles

${ }^{b)}$ Assistant Researcher, Civil and Environmental Engineering Dept., University of California, Los Angeles
} 
One such Equipment Site with a focus on field testing and monitoring of structural performance has been developed at the University of California, Los Angeles (nees@UCLA). Thenees@UCLA equipment site provides state-of-the-art equipment for forced vibration testing and seismic monitoring of full-scale structural and geotechnical systems. The equipment portfolio includes shakers for exciting structural systems, numerous sensors for monitoring accelerations and deformations within the excited structure (e.g., accelerometers and strain gauges), and real-time data acquisition and dissemination capabilities.

The major equipment components of the site are illustrated in Figure 1 and include the following:

A. Eccentric mass shakers that can apply harmonic excitation across a wide frequency range in one or two horizontal directions. These shakers can induce weak to strong forced vibration of structures. For small structures, excitation into the nonlinear range is possible when the shakers are operated near their maximum force capacity. The shakers can be operated in a wired or wireless mode.

B. Linear inertial shaker that can apply broadband excitation at low force levels. This shaker can be programmed to approximately reproduce the seismic structural response that would have occurred for any specified base-level acceleration time history (assuming the properties of the structure are known). The shaker can be controlled in a wired or wireless mode.

C. Above-ground sensors that can be installed at the ground surface or on building, bridge, or geo-structures to record acceleration or deformation responses. Accelerations are recorded with uni-directional or triaxial accelerometers. Deformations (i.e., relative displacements between two points) are recorded with LVDTs or using fiber-optic sensors.

D. Retrievable subsurface accelerometers (RSAs) that can be deployed below-ground to record ground vibrations in three directions. The sensors and their housing are specially designed to be retrievable upon the completion of testing.

E. Wireless field data acquisition system that efficiently transmits data in wireless mode from the tested structure to the high performance mobile network (see following item). 
F. High performance mobile network that (a) receives and locally stores data at a mobile command center deployed near the test site; (b) transmits selected data in near real time via satellite to the UCLA global backbone; and (c) broadcasts data via the NEESpop server into the NEESgrid for teleobservation of experiments.

As shown in Fig. 1, a typical application of the equipment would have shakers installed on or within a structure, a dense array of sensors throughout the structure and RSAs deployed below the ground surface. Data from the building sensors and RSAs are transmitted wireless via field data loggers to the mobile command center where all data are locally stored. Selected data channels and video streams could be transmitted via satellite to the UCLA global backbone for subsequent dissemination via NEESpop for teleobservation of the experiment.

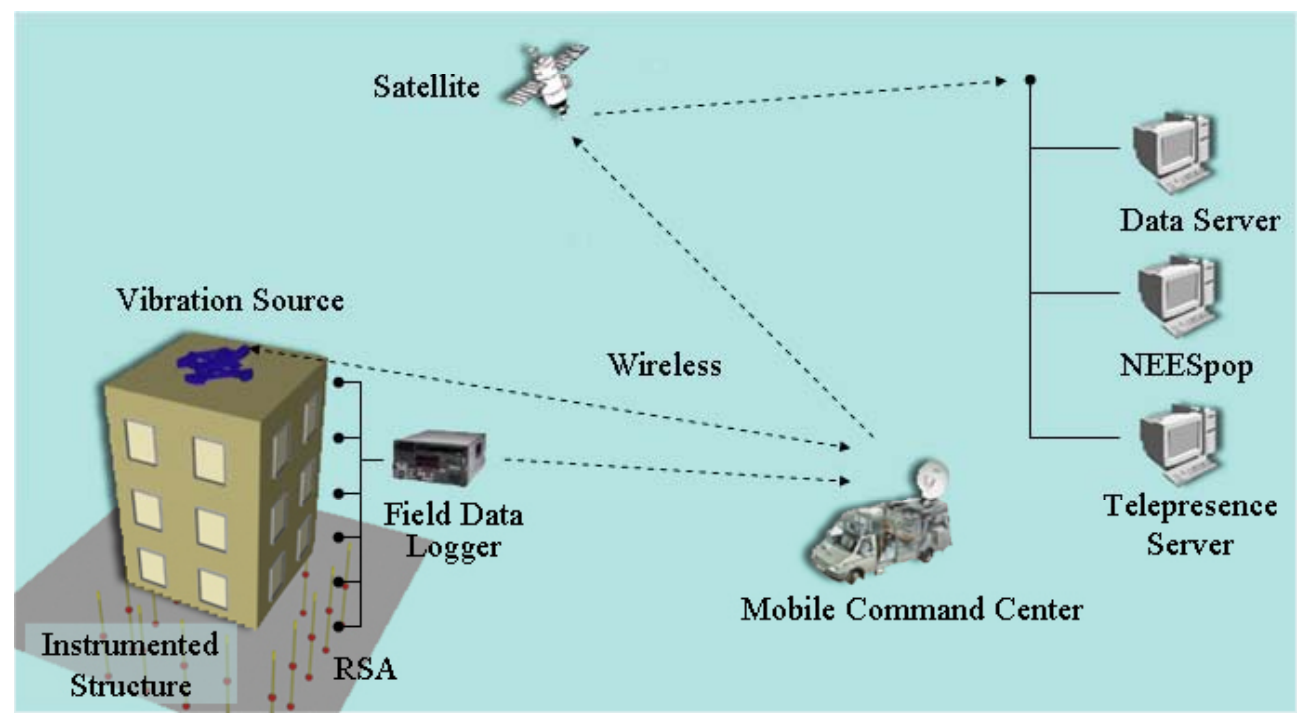

Fig. 1. Schematic illustration of deployed equipment from the nees@UCLA Site

One point that should be emphasized is that the nees@UCLA equipment can be utilized with several types of vibration sources. Obviously, the eccentric mass shakers and linear inertial shaker are two such types, but the equipment is also ideally suited for seismic monitoring of structural or geo-systems (i.e., aftershock or microtremor sources).

We anticipate several general categories of application for the nees@UCLA equipment site, including (1) building or bridge structural response/performance studies; (2) seismic health monitoring and sensor network studies; (3) response/performance studies for geostructures and soil deposits; and (4) soil-structure interaction (SSI) studies. The remainder of 
this paper briefly describes opportunities for SSI research with the nees@UCLA equipment portfolio.

\section{SOIL-STRUCTURE INTERACTION RESEARCH OPPORTUNTIES}

\section{PREVIOUS TESTING}

A principal goal of soil-structure interaction testing is often the evaluation of effective stiffness and damping associated with foundation-soil interaction, which is often quantified by so-called foundation impedance functions. Table 1 presents a summary of forced vibration tests that have been performed in the field with the objective of identifying foundation impedance functions. It should be noted that laboratory-scale studies of SSI for shallow foundations have also been performed (e.g., Gajan et al., 2004). Such tests can also provide valuable insight, but the limited scale of the models precludes the tests from properly capturing important SSI phenomena such as radiation damping.

Table 1. Summary of forced vibration tests used to infer impedance functions

\begin{tabular}{|c|c|c|c|c|c|c|c|c|c|}
\hline \multirow[b]{2}{*}{\begin{tabular}{|l|} 
Foundation \\
Dimensions
\end{tabular}} & \multirow[b]{2}{*}{$\begin{array}{c}\text { Embed. } \\
(\mathrm{m})\end{array}$} & \multirow[b]{2}{*}{$\mathrm{f}_{1}(\mathrm{~Hz})^{1}$} & \multirow[b]{2}{*}{$V_{s}(m / s)$} & \multicolumn{2}{|c|}{ Excitation } & \multicolumn{4}{|c|}{ Results } \\
\hline & & & & Source & Freq $(\mathrm{Hz})$ & $f / \widetilde{f}$ & $\begin{array}{c}\text { Impedance } \\
\text { obtained }\end{array}$ & $\begin{array}{l}\text { Freq range } \\
(\mathrm{Hz})\end{array}$ & Reference \\
\hline $3 m \times 3 m$ & $0-1.5$ & 17.5 & 305 & \begin{tabular}{|c|} 
Vibrator \\
on ground
\end{tabular} & $7-70$ & $\begin{array}{c}1.3(\mathrm{e}=1.5) ; \\
1.5(\mathrm{e}=0)\end{array}$ & $\begin{array}{c}k_{u}, c_{u}, k_{\theta}, \\
c_{\theta}\end{array}$ & $\begin{array}{l}\text { modal freq. } \\
\text { only }\end{array}$ & $\begin{array}{l}\text { Lin and Jennings, } \\
1984\end{array}$ \\
\hline $25 m \times 25 m$ & $4-5.5$ & $\begin{array}{l}\text { NS: } 2.16 ; \\
\text { EW: } 1.26\end{array}$ & 300 & $\begin{array}{l}\text { Vibrator } \\
\text { on roof }\end{array}$ & $\begin{array}{l}\text { NS: 0.8- } \\
\text { 2.5; EW: } \\
0.8-1.75\end{array}$ & $\begin{array}{c}\text { NS:1.06; } \\
\text { EW:1.1 }\end{array}$ & $\begin{array}{c}k_{u}, c_{u}, k_{\theta}, \\
c_{\theta}\end{array}$ & $\begin{array}{l}\text { NS:0.8-2.5; } \\
\text { EW:0.8-1.75 }\end{array}$ & $\begin{array}{l}\text { Luco et al., 1988; } \\
\text { Wong et al., } 1988\end{array}$ \\
\hline $\begin{array}{l}1.3 \mathrm{~m} \times 1.3 \mathrm{~m} ; \\
1.2 \mathrm{~m} \times 1.1 \mathrm{~m}\end{array}$ & 0 & $n / a$ & $120 ; 75$ & $\begin{array}{l}\text { Vibrator } \\
\text { on fndn. }\end{array}$ & $10-60$ & $\mathrm{n} / \mathrm{a}$ & $\begin{array}{c}k_{u}, c_{u}, k_{\theta}, \\
c_{\theta}\end{array}$ & $0-60$ & $\begin{array}{l}\text { Crouse et al., } \\
1990\end{array}$ \\
\hline $\mathrm{d}=10.8 \mathrm{~m}$ & 5.2 & 9.37 & 300 & $\begin{array}{c}\text { Vibrator } \\
\text { on } \\
\text { roof/fndn }\end{array}$ & $2-20$ & 2 & $\begin{array}{c}k_{u}, c_{u}, k_{\theta}, \\
c_{\theta}, k_{u}, \\
c_{u \theta}, k_{v}, c_{v}\end{array}$ & $5-14$ & $\begin{array}{l}\text { DeBarros and } \\
\text { Luco, } 1995\end{array}$ \\
\hline
\end{tabular}

Testing by Lin and Jennings (1984) was performed on a small model structure and provided spring and dashpot coefficients near the fundamental-mode system frequency. The results were used to evaluate the effect of foundation embedment on the impedance functions. Comparisons to impedance function models for unembedded foundations were favorable, but a bias was noted for embedded foundations.

Testing by Luco et al. (1988) was performed on a full-scale structure (i.e., the 9-story Millikan Library on the Caltech campus). The results were used to evaluate whether simple impedance function models could reproduce the observed foundation impedance functions. Example results are shown in Figure 2 for normalized translation and rocking impedance 
functions. The "rigid" and "flexible" experimental results relate to the use of different sensors installed on the foundation for system identification. Normalized results are plotted in Figure 2 against the predictions of the Apsel and Luco (1987) theoretical impedance function model. There are no significant differences between the results for the "flexible" and "rigid" foundation assumptions. The results for rocking (left frame) compare favorably to the predictions from theory, while the results for translation (right frame) do not.

Testing by Crouse et al. (1990) was performed on foundation pads for typical accelerograph installations. Tested foundations were $\sim 1.2 \mathrm{~m}$ square concrete pads with and without corner piers. Adjustments to the site shear wave velocity were needed to match the observed impedance functions.
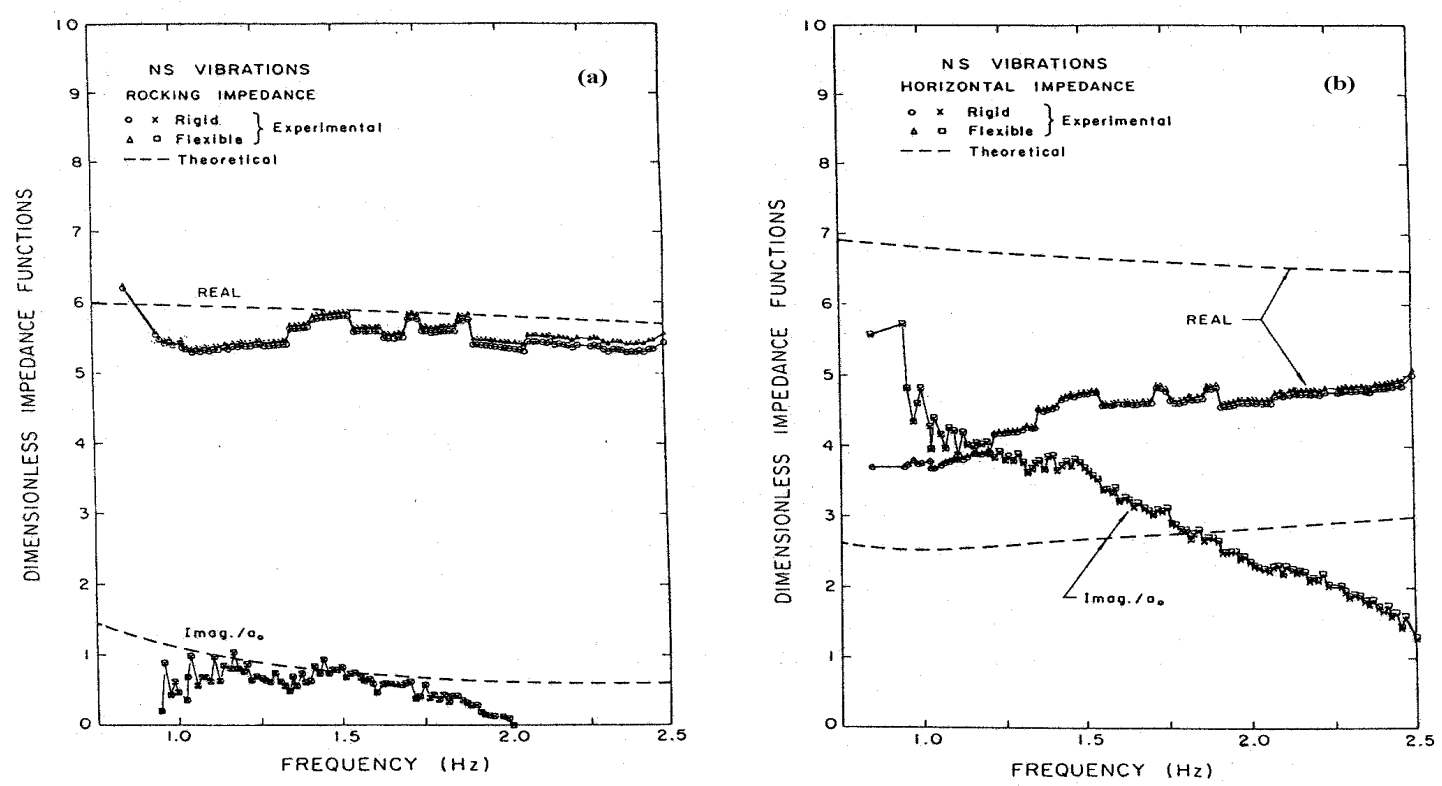

Fig. 2. Comparison of theoretical and experimental estimates of impedance functions for Millikan library building, NS direction (after Wong et al., 1988)

Testing by deBarros and Luco (1995) was performed on the one-quarter scale reinforced concrete Hualien, Taiwan containment model. The model structure is of a similar design to the well-known Lotung, Taiwan containment model, but is sited on foundation soils with considerably larger shear stiffness than those at Lotung $\left(\mathrm{V}_{\mathrm{s}} \approx 300 \mathrm{~m} / \mathrm{s}\right.$ at Hualien vs. $\approx 100$ $150 \mathrm{~m} / \mathrm{s}$ at Lotung). As with the Millikan library, results compared to theory more favorably for rocking than for horizontal translations. Data interpretation was complicated by differences in the results for perpendicular horizontal directions, which was attributed to laterally heterogeneous soil properties. 
Attempts to evaluate impedance from seismic data have been made by Moslem and Trifunac (1986) and Kim (2001). The results for rocking stiffness were meaningful only near the first-mode frequency. The identification exercises were not particularly successful for translational stiffness nor for damping in either translation or rocking due to noise effects and potential synchronization problems in the data acquisition systems (i.e., small time lags can produce significant errors in measured damping). An interesting finding from the Moslem and Trifunac (1986) work was that the rocking stiffness during earthquake shaking was reduced from that during forced vibration testing, which was attributed to soil nonlinearity.

\section{OPPORTUNITIES FOR FUTURE FOUNDATION TESTING}

Several pertinent observations can be made from the testing/analysis in the literature. First, investigators often found that linking the site shear wave velocity profile to the velocity that provides the best match to the field data to be non-trivial. This highlights both the difficulty of fitting simplified theoretical models to field measurements and the consequent need for an inventory of test data to guide parameter selection. Second, the results from the Millikan library highlight the importance of soil nonlinearity on foundation-soil stiffness. Unfortunately, these test data were not sufficient to define the soil strain field beneath the foundation, which is needed to enable rational predictions of nonlinear soil properties within this domain. Third, the available data generally does not address, or provides very limited coverage, of a number of significant issues including: (1) localized foundation settlement induced by cyclic loading, which has been widely observed following earthquakes (e.g., see Bray and Stewart, 2000); and (2) foundation-soil damping associated with soil hysteresis or radiation effects.

The nees@UCLA equipment is ideally suited for meeting these research needs. Whether employed on existing structures or specially constructed model structures in the field, the equipment could be used to:

1. Dynamically excite the structure with eccentric mass shakers or the linear inertial shaker, thus creating base shear forces and moments, which would lead to relative foundation/free-field displacements and rocking;

2. Record foundation and ground vibrations with accelerometers, displacement transducers, and/or strain gauges, which quantify foundation vibrations and potential foundation deformations. 
3. Record subsurface accelerations with RSAs, thus enabling recordings of the wave field emanating from the foundation and the inference of subsurface shear strains.

4. Transmit the data wireless to the mobile command center, where it can be locally stored and viewed, and also sent via satellite to the UCLA global backbone for dissemination over the internet.

Accordingly, foundation vibration testing is expected to be a major application of the nees@UCLA equipment site once it becomes operational in October 2004.

\section{CONCLUSIONS}

The development of the NSF-funded nees@UCLA Equipment Site is approaching completion, and will provide a valuable shared-use resource for field testing and monitoring of structural and geotechnical performance. The nees@UCLA equipment portfolio includes shakers for exciting structural systems, numerous sensors for monitoring accelerations and deformations within the excited structure (e.g., accelerometers and strain gauges), and realtime data acquisition and dissemination capabilities. The full nees@UCLA site goes "on line" for public use October 1, 2004. The site provides equipment that is ideally suited to soil-structure interaction testing and research.

\section{ACKNOWLEDGEMENTS}

This research was funded by the National Science Foundation under Cooperative Agreement No.CMS-0086596, and is gratefully acknowledged.

\section{REFERENCES}

Apsel, R.J. and Luco, J.E., 1987. Impedance functions for foundations embedded in a layered medium: an integral equation approach, J. Earthquake Engrg. Struct. Dynamics 15, 213-231.

Bray, J.D. and Stewart, J.P., coordinators, 2000. Chapter 8: Damage patterns and foundation performance in Adapazari. Kocaeli, Turkey Earthquake of August 17, 1999 Reconnaissance Report, T.L. Youd, J.P. Bardet, and J.D. Bray, eds., Earthquake Spectra 16, Supplement A, 163189.

Gajan, S. Phalen, J.D., Kutter, B.L., Hutchinson, T.C., and Martin, G.R., 2004. Centrifuge modeling of nonlinear cyclic load-deformation behavior of shallow foundations, Proc. $11^{\text {th }}$ Int. Conf. Soil 


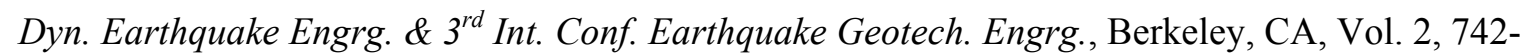
749.

Crouse, C.B., Hushmand, B., Luco, J.E., and Wong, H.L., 1990. Foundation impedance functions: Theory versus Experiment, J. Geotech. Engrg. 116, 432-449.

de Barros, F.C.P. and Luco, J.E., 1995. Identification of foundation impedance functions and soil properties from vibration tests of the Hualien containment model, J. Soil Dyn. Earthquake Eng. $14,229-248$.

Kim, S., 2001. Calibration of simple models for seismic soil structure interaction from field performance data, Ph.D. Dissertation, Univ. of Calif., Los Angeles.

Lin, A.N. and Jennings, P.C., 1984. Effect of embedment on foundation-soil impedances, J. Engrg. Mech.. 110, 1060-1075.

Luco, J.E., Trifunac, M.D., and Wong, H.L., 1988. Isolation of soil-structure interaction effects by full-scale forced vibration tests, J. Earthquake Engrg. Struct. Dynamics 16, 1-21.

Moslem, K. and Trifunac, M.D., 1986. Effects of soil-structure interaction on the response of building during the strong earthquake ground motion, Rpt. No. 86-04, Univ. of Southern California, Dept. of Civil Engrg.

Wong, H.L., Trifunac, M.D., and Luco, J.E., 1988. A comparison of soil-structure interaction calculations with results of full-scale forced vibration tests, Soil Dyn. \& Earthquake Engrg. 7, 2231. 